\title{
Setiburst: A Robotic, Commensal, Realtime Multi-Science Backend For The Arecibo Telescope
}

Jayanth Chennamangalam

David MacMahon

Jeff Cobb

Aris Karastergiou

Andrew P. V. Siemion

See next page for additional authors

Follow this and additional works at: https://researchrepository.wvu.edu/faculty_publications

\section{Digital Commons Citation}

Chennamangalam, Jayanth; MacMahon, David; Cobb, Jeff; Karastergiou, Aris; Siemion, Andrew P. V.; Rajwade, Kaustubh; Armour, Wes; Gajjar, Vishal; Lorimer, Duncan R.; and McLaughlin, Maura A., "Setiburst: A Robotic, Commensal, Realtime Multi-Science Backend For The Arecibo Telescope" (2017). Faculty \& Staff Scholarship. 670.

https://researchrepository.wvu.edu/faculty_publications/670

This Article is brought to you for free and open access by The Research Repository @ WVU. It has been accepted for inclusion in Faculty \& Staff Scholarship by an authorized administrator of The Research Repository @ WVU. For more information, please contact beau.smith@mail.wvu.edu. 


\section{Authors}

Jayanth Chennamangalam, David MacMahon, Jeff Cobb, Aris Karastergiou, Andrew P. V. Siemion, Kaustubh Rajwade, Wes Armour, Vishal Gajjar, Duncan R. Lorimer, and Maura A. McLaughlin 


\title{
SETIBURST: A Robotic, Commensal, Realtime Multi-science Backend for the Arecibo Telescope
}

\author{
Jayanth Chennamangalam ${ }^{1}$, David MacMahon ${ }^{2}$, Jeff $\mathrm{Cobb}^{2}$, Aris Karastergiou ${ }^{1,3,4}$, Andrew P. V. Siemion ${ }^{2,5,6}$, Kaustubh Rajwade ${ }^{7}$, \\ Wes Armour $^{8}$, Vishal Gajjar ${ }^{2}$, Duncan R. Lorimer ${ }^{7,9}$, Maura A. McLaughlin ${ }^{7}$, Dan Werthimer ${ }^{2}$, and Christopher Williams ${ }^{1}$ \\ ${ }^{1}$ Astrophysics, University of Oxford, Denys Wilkinson Building, Keble Road, Oxford OX1 3RH, UK; jayanth@astro.ox.ac.uk \\ ${ }^{2}$ Department of Astronomy, University of California Berkeley, Berkeley, CA 94720, USA \\ ${ }^{3}$ Department of Physics and Electronics, Rhodes University, P.O. Box 94, Grahamstown 6140, South Africa \\ ${ }^{4}$ Physics Department, University of the Western Cape, Cape Town 7535, South Africa \\ 5 ASTRON, P.O. Box 2, 7990 AA Dwingeloo, The Netherlands \\ ${ }^{6}$ Department of Astrophysics, Radboud University, P.O. Box 9010, 6500 GL Nijmegen, The Netherlands \\ ${ }^{7}$ Department of Physics and Astronomy, West Virginia University, P.O. Box 6315, Morgantown, WV 26506, USA \\ ${ }^{8}$ Oxford e-Research Centre, University of Oxford, Keble Road, Oxford OX1 3QG, UK \\ ${ }^{9}$ National Radio Astronomy Observatory, P.O. Box 2, Green Bank, WV 24944, USA \\ Received 2016 May 11; revised 2017 January 10; accepted 2017 January 12; published 2017 February 14
}

\begin{abstract}
Radio astronomy has traditionally depended on observatories allocating time to observers for exclusive use of their telescopes. The disadvantage of this scheme is that the data thus collected is rarely used for other astronomy applications, and in many cases, is unsuitable. For example, properly calibrated pulsar search data can, with some reduction, be used for spectral line surveys. A backend that supports plugging in multiple applications to a telescope to perform commensal data analysis will vastly increase the science throughput of the facility. In this paper, we present "SETIBURST," a robotic, commensal, realtime multi-science backend for the $305 \mathrm{~m}$ Arecibo Telescope. The system uses the $1.4 \mathrm{GHz}$, seven-beam Arecibo $L$-band Feed Array (ALFA) receiver whenever it is operated. SETIBURST currently supports two applications: SERENDIP VI, a SETI spectrometer that is conducting a search for signs of technological life, and ALFABURST, a fast transient search system that is conducting a survey of fast radio bursts (FRBs). Based on the FRB event rate and the expected usage of ALFA, we expect 0-5 FRB detections over the coming year. SETIBURST also provides the option of plugging in more applications. We outline the motivation for our instrumentation scheme and the scientific motivation of the two surveys, along with their descriptions and related discussions.
\end{abstract}

Key words: extraterrestrial intelligence - instrumentation: miscellaneous - pulsars: general

\section{Introduction}

Radio astronomy relies on observations for which telescope time is obtained following a competitive proposal review. This process is critical because telescope time is limited: only one kind of observation can usually be done at a given time. In addition to this exclusivity, the utility of the collected data is usually restricted to the specific kind of experiment that it was obtained for. Data reuse within a given field is standard practice - e.g., the original fast radio burst (FRB; see Section 1.2) was discovered in a reprocessing of data from a fast radio transient survey of the Magellanic Clouds using the $64 \mathrm{~m}$ Parkes Radio Telescope (Lorimer et al. 2007) - but cross-disciplinary data reuse is a rarity. For example, spectral line surveys, due to long integration times used in its observations, result in data products that cannot be reused in a search for pulsars. On the other hand, properly calibrated pulsar search data can be used for spectral line surveys as well, but this is rarely done. This severely restricts the science throughput of a facility. To optimize data collection and analysis, commensal observing is increasingly being employed, wherein multiple data processing/recording processes run simultaneously on data from the telescope during observations. In such a scheme, telescope pointing remains under the control of the primary observer, but secondary observers also have access to data, vastly increasing the available sky coverage.

Commensal observing was pioneered by the early searches for extraterrestrial intelligence (SETI) at the Hat Creek Radio
Observatory (Bowyer et al. 1983), and later, at the Arecibo Observatory, home to the $305 \mathrm{~m}$ diameter Arecibo telescope. The original need for commensal observing was due to the fact that SETI requires searching a large parameter space for which a significant amount of telescope time is required, and the inability of allocating dedicated time to such a large survey that is speculative in nature. The Search for Radio Emissions from Nearby Developed Intelligent Populations (SERENDIP) project at the Arecibo Observatory-of which the instrument described in this paper is a part-has, throughout its existence, relied on commensal data processing (see, for example, Bowyer et al. 1993). Technologically, in recent times, relatively inexpensive networking hardware and high-performance computing (HPC) machines have made it possible to build multiple HPC-based backends that can easily distribute and process radio telescope data, enabling commensal data processing. The Allen Telescope Array was built with commensal observing as a design goal, such that SETI and non-SETI observations could be done in parallel (Welch et al. 2009). In high time resolution astronomy, the need for commensal observing has been made apparent by the discovery of new classes of fast radio transients, such as rotating radio transients (RRATs; McLaughlin et al. 2006) and FRBs (Lorimer et al. 2007; Thornton et al. 2013). The V-FASTR experiment at the Very Large Baseline Array (Wayth et al. 2012) is a commensal search for fast transients. VLITE ${ }^{10}$

${ }^{10}$ http://vlite.nrao.edu/ 
is a 10-antenna system at the Very Large Array that performs ionospheric observations, transient searches, and imaging parallel to regular observations. Among new facilties, the Australian Square Kilometre Array Pathfinder (ASKAP) is used for the Commensal Realtime ASKAP Fast Transients survey (Macquart et al. 2010). In this paper, we describe a new instrument at the Arecibo Observatory that is centered around the idea of commensal observing, with a SETI experiment and a fast transient survey as consumers of the collected data.

The outline of this paper is as follows. In the following subsections, we introduce the two science motivations of the project, namely, SETI and fast radio transients. In Section 2, we describe the technical details of the system: the SERENDIP VI SETI backend and the ALFABURST fast transient backend. In Section 3, we describe the two commensal surveys we are undertaking, and we conclude in Section 4.

\subsection{SETI}

The quest for life in the universe has seen much progress in recent years, with the exploration of the solar system and the detection of a large number of extrasolar planets. A whole new field-astrobiology-has emerged to tackle the problem of whether life exists elsewhere in the Galaxy. SETI aims one step further, to answer the more challenging question of the existence of technological intelligent life. One of the first SETI attempts followed the suggestion by Cocconi \& Morrison (1959) that ETI may transmit narrow-band beacons near the radio emission line of neutral hydrogen, at $1420 \mathrm{MHz}$, enabling radio astronomers in other civilizations to detect them. Radio SETI observations started with Drake (1961) who searched for narrow-band lines and have continued to the present day with increasing levels of sophistication. For instance, Siemion et al. (2013) recently searched for interplanetary radar signals in multi-planet systems during conjunctions, ${ }^{11}$ and established that $\lesssim 1 \%$ of transiting exoplanet systems host civilizations that emit narrow-band radiation in the $1-2 \mathrm{GHz}$ band with an equivalent isotropically radiated power (EIRP) of $\sim 1.5 \times 10^{13} \mathrm{~W}$.

Whether ETI would set up beacons for the benefit of curious radio astronomers in other civilizations is unknown, but setting up such beacons is the best possible way to advertise our presence in the universe. Radio emission is energetically, and hence, economically inexpensive to generate. Radio waves can travel vast distances with relatively less attenuation due to the interstellar medium (ISM) compared to electromagnetic radiation at other wavelengths, ensuring a better likelihood of signal reception. Cleverly designed beacons, such as extremely narrow-band signals near a natural emission line commonly used in studying the Galaxy, such as that proposed by Cocconi \& Morrison (1959), will increase the likelihood of the signal being noticed by radio astronomers elsewhere. The rationale behind searching for extremely narrow-band signals is that the narrowest astrophysical lines are of the order of hundreds of $\mathrm{Hz}$ wide. The narrowest line detected has a width of $550 \mathrm{~Hz}$ (Cohen et al. 1987). Even if a civilization does not set up a beacon, radio emission created by their technology could leak out into space, at least during the early stages of their technological development, in much the same way as coherent

\footnotetext{
${ }^{11}$ Note the error in the sensitivity calculation in Siemion et al. (2013) and the values reported in their Table 3: their characteristic sensitivity should be $\sim 2 \times 10^{-22} \mathrm{erg} \mathrm{s}^{-1} \mathrm{~cm}^{-2}$, and the exponent in footnote (d) in their Table 3 should be -22 .
}

radio emission produced quite commonly by human technology routinely leaks out to space. Given that humans have been transmitting in the radio for more than a century, the earliest radio transmissions have traversed a distance $>30 \mathrm{pc}$ away from us. It is conceivable that leakage signals from other civilizations may be picked up on Earth; though, given the fact that they are not intentional transmissions to us, it is unlikely that they would be easy to detect amid the background noise.

\subsection{Fast Radio Bursts}

Fast radio transients have been a staple of radio astronomy research for decades, starting with the discovery of the first pulsars (Hewish et al. 1968). In recent years, new classes of such transients have emerged, namely, RRATs, which are thought to be highly intermittent pulsars (McLaughlin et al. 2006), and FRBs (Lorimer et al. 2007; Thornton et al. 2013). However, in spite of the long history and recent discoveries, robotic surveys in the radio are only beginning to be employed. For example, the Survey for Pulsars and Extragalactic Radio Bursts ${ }^{12}$ at the Parkes telescope uses the "Heimdall" realtime data processing pipeline. A robotic system in the radio that operates as long as a supported receiver is available can radically increase the time available on the sky and lead to discoveries of fast transients such as RRATs and FRBs. Realtime detection has the advantage of being able to trigger other telescopes that are geographically separated and operate at other frequencies, as exemplified by Keane et al. (2016), whereas offline analysis of data usually results in a latency of days to months. The ability to follow-up detected events within hours or days is critical to the identification of potential afterglows or other indicators of the same event at different wavelengths, helping shed light on the location of, and the physics behind, these exotic sources.

FRBs are broadband radio pulses with observed widths of the order of a few milliseconds. Due to the nature of the dispersion caused by the ionized ISM, lower frequency components of the pulse are delayed much more than the higher frequency components. This delay is quantified in terms of the dispersion measure (DM), which, for FRBs, is greater than that contributed by the ISM of the Galaxy, indicating an extragalactic origin. The millisecond timescale of the pulse implies a compact object progenitor. Due to the fact that all reported FRBs have been detected using single-dish radio telescopes that have wide beams, localization, and hence, association with sources at other wavelengths, has proven to be a challenge. Therefore, a conclusive explanation of what FRBs are has remained elusive, with various theories being proposed. Extragalactic-origin theories that posit cataclysmic explosions include the gravitational collapse of supramassive neutron stars (Falcke \& Rezzolla 2014) and binary neutron star mergers (Totani 2013). Extragalactic-origin theories that predict repetition include giant-pulse-emitting pulsars (see, for example, Lyutikov et al. 2016), flaring magnetars (Popov \& Postnov 2013, for instance), and Alfvén wings around planetary companions to pulsars (Mottez \& Zarka 2014). There is at least one Galactic-origin theory (that also predicts pulse repetition) wherein these bursts originate in flare stars, and are dispersed by the stellar corona (Loeb et al. 2014). A resolution of the mystery has been made complicated by two recent discoveries, namely, that of FRB 150418, which has been claimed to be

\footnotetext{
12 https://sites.google.com/site/publicsuperb/
} 
associated with a slow radio transient in an elliptical galaxy interpreted to be an afterglow of a cataclysmic, non-repeating event (Keane et al. 2016), and that of FRB 121102, which has been shown to repeat (Spitler et al. 2014, 2016). ${ }^{13}$

Eighteen FRBs have been reported in the published literature $^{14}$ (Petroff et al. 2016). All reported FRBs except two were discovered using the Parkes telescope. The exceptions were discovered using the Arecibo telescope (Spitler et al. 2014) and the Green Bank Telescope (Masui et al. 2015). Since FRBs are non-repeating/highly intermittent, increasing the amount of observing time available will allow more to be detected, enabling a better understanding of these events.

\subsection{SETIBURST}

Keeping in mind the aforementioned scientific motivations, we have designed, developed, and deployed SETIBURST, an automated, commensal, realtime multi-science backend for the Arecibo telecope. SETIBURST has two plug-in applications: SERENDIP VI (referred to as "S6" henceforth), the latest in the SERENDIP series of SETI spectrometers, and ALFABURST, a fast transient search pipeline. SETIBURST performs commensal processing of signals from the $1.4 \mathrm{GHz}$ seven-beam Arecibo $L$-band Feed Array ${ }^{15}$ (ALFA) receiver. ALFA is Arecibo's workhorse survey receiver, being used for such large-scale surveys as the Pulsar ALFA (PALFA) survey (Cordes et al. 2006), which has so far resulted in the discovery of 145 pulsars and one FRB (Cordes et al. 2006; Spitler et al. 2014; Lazarus et al. 2015); the Galactic ALFA Continuum Transit Survey (GALFACTS; see, for example, Taylor \& Salter 2010); and the Arecibo Galaxy Environment Survey (AGES; see, for example, Auld et al. 2006). ALFA is well-suited for an FRB survey at Arecibo given that all except the Green Bank FRB were detected at $1.4 \mathrm{GHz}$. Being a survey receiver with multiple beams, ALFA is suited for SETI surveys as well.

\section{System Description}

SETIBURST is a heterogeneous instrument, i.e., it uses Field Programmable Gate Arrays (FPGAs) and HPC machines equipped with Graphics Processing Units (GPUs). Heterogeneous instruments have increased in popularity in astronomical signal processing applications in recent years (see, for example, DuPlain et al. 2008; Woods 2010) because they combine the high-bandwidth capabilities of FPGAs with the features, flexibility, and ease of programming of GPUs. The network switch has the potential to act as a data hub into which HPC backends may be plugged in, enabling multiple simultaneous experiments.

Figure 1 shows the high-level architecture of the system. The digital system processes signals from the ALFA receiver. ALFA is a seven-beam system that operates in the $1225-1525 \mathrm{MHz}$ range, with the seven beams arranged in a hexagonal pattern. Each beam is approximately 3!5 wide. The receiver has a cold sky system temperature of $\sim 30 \mathrm{~K}$. The central beam has a gain of $\sim 11 \mathrm{~K} \mathrm{Jy}^{-1}$, with the peripheral beams having a slightly lower gain. Some of the sidelobes of the ALFA beams are sensitive as well, with the peak of these

\footnotetext{
${ }^{13}$ In developments published during the late stages of the review process of this manuscript, the repeating source FRB 121102 has been shown to be associated with an extragalactic source (Chatterjee et al. 2017).

${ }^{14}$ http://www.astronomy.swin.edu.au/pulsar/frbcat/

15 http://www.naic.edu/alfa/
}

sidelobes having a loss of only $-8.5 \mathrm{~dB}$, i.e., the gain at the sidelobe peak is only (1/7)th of that at boresight of the central beam. The advantage of this is that it has the effect of increasing the area on the sky, and the disadvantages include increased uncertainty in localization and poor fidelity in the measurement of the spectral indices of FRBs that may have been picked up in these sidelobes (Spitler et al. 2014). S6 uses $280 \mathrm{MHz}$ of ALFA's $300 \mathrm{MHz}$ bandwidth, while ALFABURST, in its current version, supports a bandwidth of $56 \mathrm{MHz}$.

\subsection{FPGA Gateware}

The 14 intermediate frequency (IF) signals from ALFA (one per polarization per beam) are split before being distributed to various backends at the observatory. SETIBURST hardware taps into these split signals, and digitizes them. The hardware consists of two ROACH $2{ }^{16}$ FPGA boards, each equipped with two 1 Gsps ADC16x250-8 ${ }^{17}$ analog-to-digital converters (ADCs). The first board (denoted by "ROACH2 A" in Figure 1) processes beams 0 through 3, while the second ROACH2 board ("ROACH2 B") processes beams 4 through 6. The ADCs first sample the data at $896 \mathrm{MHz}$ and digitizes it to 8 bits. The FPGA gateware uses a polyphase filterbank (PFB) to channelize the data to 4096 channels, with a resulting time resolution of $9.143 \mu \mathrm{s}$. The PFB is implemented using the standard CASPER ${ }^{18}$ blocks pfb_fir_real ${ }^{19}$ and fft_wideband_real. ${ }^{20}$ The prefiltering uses four taps, and the coefficients are the product of a sinc function and a Hamming window.

The bandpass is split into eight sub-bands, which are packetized separately, each addressed to a different HPC pipeline, and transmitted over 10 Gigabit Ethernet $(10 \mathrm{GbE})$. The packetization mechanism assigns a different IP address to packets sent to different HPC nodes, using IP addresses stored in software registers on the $\mathrm{ROACH} 2$ boards, that are programmable at run-time. Each HPC node runs two software instances/pipelines for all beams and polarizations (see Section 2.2). Even though our digitization scheme results in a bandwidth of $448 \mathrm{MHz}$, ALFA has a bandwidth of only $300 \mathrm{MHz}$. To remove channels with no information and to reduce the output data rate, we pare the band down to 2560 channels (corresponding to a bandwidth of $280 \mathrm{MHz}$ ) and these are packetized. The complex samples at the output of the PFB are packetized into 1296 byte long User Datagram Protocol (UDP) packets. Figure 2 shows the S6 packet format. Each packet contains an 8 byte header that contains a 48 bit spectrum counter, a 12 bit field indicating the first channel in the packet (denoted by $P$ in Figure 2), and a 4 bit beam identifier that takes on values in the range of $0-6$ (denoted by $B$ in Figure 2 ). The spectrum count is used at the receiver (HPC) for packet loss checking. Each packet also consists of a 64 bit footer that contains a 32 bit cyclic redundancy check for error detection on the HPC. The bytes that make up these packets are transmitted in network byte order.

\footnotetext{
16 "Reconfigurable Open Architecture Computing Hardware 2"; http://casper. berkeley.edu/wiki/ROACH2.

17 http://casper.berkeley.edu/wiki/ADC16x250-8

18 Collaboration for Astronomy Signal Processing and Electronics Research: https://casper.berkeley.edu.

19 https://casper.berkeley.edu/wiki/Pfb_fir_real

20 https://casper.berkeley.edu/wiki/Fft_wideband_real
} 


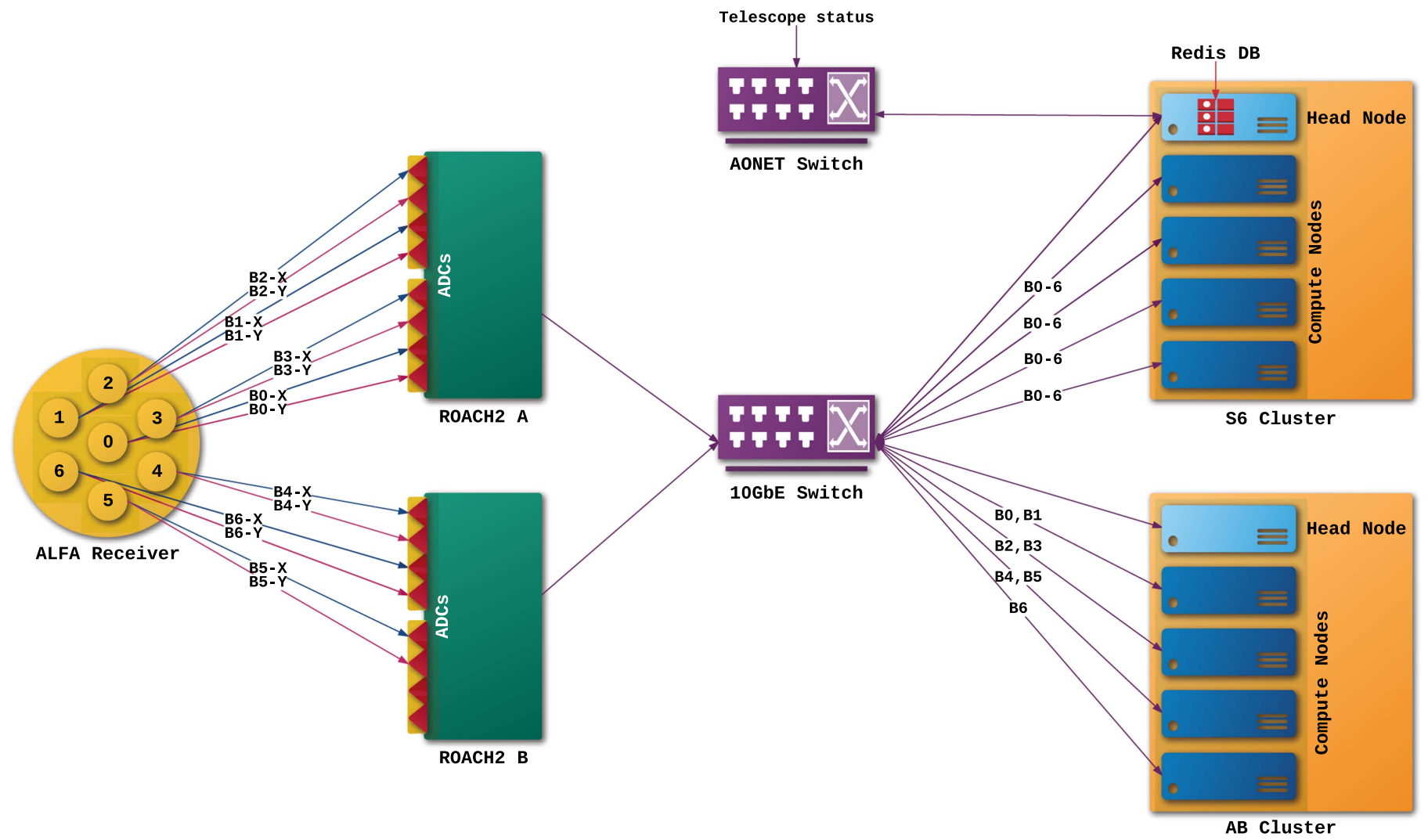

Figure 1. Simplified high-level architecture of the SETIBURST system. Both polarizations (denoted by $X$ and $Y$ ) of all ALFA beams (denoted by $B 0-B 6$ ) are processed by two ROACH2 FPGA boards and distributed to compute nodes through a $10 \mathrm{GbE}$ swith. "AB" stands for ALFABURST in this diagram. The setup is described in detail in Section 2.

For ALFABURST, polyphase channelization is followed by computation of pseudo-Stokes values $X X^{*}, Y Y^{*}, \operatorname{Re}\left(X Y^{*}\right)$, and $\operatorname{Im}\left(X Y^{*}\right)$ (each 16 bits wide), where $X$ and $Y$ are the Fourier representations of the two polarizations, and $X^{*}$ and $Y^{*}$ are their respective complex conjugates. Note that full-Stokes values can be computed from these values. The spectra are then timeintegrated by a factor of 14 , with a resulting time resolution of $128 \mu \mathrm{s}$. The spectra are then packetized into UDP packets, and transmitted over $10 \mathrm{GbE}$. To conform to the Ethernet "jumbo frame" standard, each packet needs to be no more than 9000 bytes long. Therefore, for each beam, the 4096-channel spectrum is split into four sub-bands, each containing 1024 channels, with one sub-band per packet. Figure 3 shows the ALFABURST packet format. In addition to the data, the UDP payload also contains a 64 bit header that includes a 48 bit integration count, a one-byte sub-band identifier that takes on values in the range of $0-3$ (denoted by $S$ in Figure 3), and a one-byte beam identifier that takes on values in the range of $0-6$ (denoted by $B$ in Figure 3). The integration count, along with the sub-band identifier allows us to check for missing packets on the receiving (HPC) side. The integration count, along with a timestamp of when that count was reset to zerowhich is read from elsewhere by the HPC software-allows us to get the timestamp of each packet. Each packet also consists of a footer similar to the one in S6. As in the case of S6, bytes are transmitted in network byte order.

\subsection{SERENDIP VI Software}

The UDP packets that are transmitted by the FPGA are forwarded to appropriate nodes in the HPC cluster by a Juniper
Networks EX4500-LB $10 \mathrm{GbE}$ switch. The S6 HPC cluster consists of five server-class computers-one "head node," and four "compute nodes," as shown in Figure 1. Each compute node is equipped with two Mellanox MCX312A-XCBT $10 \mathrm{GbE}$ network interface cards (NICs) that receive data from the $10 \mathrm{GbE}$ switch. Each compute node is a dual-socket, dualGPU machine equipped with RAID data disks. We use commercial gaming GPUs, namely NVIDIA GeForce GTX 780 Ti cards.

S6 uses the HASHPIPE ${ }^{21}$ software for data acquisition and processing. HASHPIPE is a multi-threaded data transport framework that moves high-bandwidth input data from the $10 \mathrm{GbE}$ NICs through a series of shared memory ring buffers and signal processing threads, all the way to writing the output to the disk. HASHPIPE is designed with a modular architecture so that user-supplied modules may be plugged in to perform certain tasks. The first module that interfaces with the NIC is the "network thread." The network thread reads data from the NIC, checks for missing packets, and writes the data to the first shared memory ring buffer. The next thread, the "GPU thread," reads that data and performs fine channelization. Each of the 4096 channels that arrive from the FPGA are channelized into 131072 channels by the GPU, with a resulting frequency resolution of $\sim 0.8 \mathrm{~Hz}$ and time resolution of $1.198 \mathrm{~s}$. This data is then written to the next shared memory ring buffer where it is read by the next thread, the "CPU thread." The CPU thread performs thresholding as follows: to estimate the mean power level of spectra, this thread boxcar averages each spectrum with a window that is 1024 channels long and computes the mean of

\footnotetext{
${ }^{21}$ High availability shared pipeline engine; available upon request.
} 


\begin{tabular}{|l|c|c|c|c|c|c|c|c|c|c|}
\hline & 0 & 1 & 2 & 3 & 4 & 5 & 6 & 7 & $\leftarrow$ Byte \\
\hline Header & \multicolumn{7}{|c|}{ Spectrum count } \\
\hline Payload 0 & $\operatorname{Re}\left(X_{0}\right)$ & $\operatorname{Im}\left(X_{0}\right)$ & $\operatorname{Re}\left(X_{1}\right)$ & $\operatorname{Im}\left(X_{1}\right)$ & $\operatorname{Re}\left(Y_{0}\right)$ & $\operatorname{Im}\left(Y_{0}\right)$ & $\operatorname{Re}\left(Y_{1}\right)$ & $\operatorname{Im}\left(Y_{1}\right)$ & \\
\hline Payload 1 & $\operatorname{Re}\left(X_{2}\right)$ & $\operatorname{Im}\left(X_{2}\right)$ & $\operatorname{Re}\left(X_{3}\right)$ & $\operatorname{Im}\left(X_{3}\right)$ & $\operatorname{Re}\left(Y_{2}\right)$ & $\operatorname{Im}\left(Y_{2}\right)$ & $\operatorname{Re}\left(Y_{3}\right)$ & $\operatorname{Im}\left(Y_{3}\right)$ & \\
\hline$\ldots$ & $\ldots$ & $\ldots$ & $\ldots$ & $\ldots$ & $\ldots$ & $\ldots$ & $\ldots$ & $\ldots$ & \\
\hline Payload 159 & $\operatorname{Re}\left(X_{318}\right)$ & $\operatorname{Im}\left(X_{318}\right)$ & $\operatorname{Re}\left(X_{319}\right)$ & $\operatorname{Im}\left(X_{319}\right)$ & $\operatorname{Re}\left(Y_{318}\right)$ & $\operatorname{Im}\left(Y_{318}\right)$ & $\operatorname{Re}\left(Y_{319}\right)$ & $\operatorname{Im}\left(Y_{319}\right)$ & \\
\hline Footer & \multicolumn{8}{|c|}{0} \\
\hline
\end{tabular}

Figure 2. SERENDIP VI packet format, as described in Section 2.1. Each UDP payload is 1296 bytes long, with an 8 byte header and an 8 byte footer. The 12 bit field denoted by $P$ indicates the first channel of this packet, and the 4 bit field denoted by $B$ contains the beam identifier. Bytes are transmitted in left-to-right, top-tobottom order, i.e., in network byte order.

the smoothed spectrum. Channels that have values 20 times the mean are considered events of interest, which we term "hits." We note that the power spectra follow a $\chi^{2}$ distribution with two degrees of freedom, such that the mean and root mean square (rms) are equal. Therefore, the ratio of the detected power in a given channel to the mean power level is a measure of the $\mathrm{S} / \mathrm{N}$ in that channel. The hits are stored as a function of time, frequency, and sky coordinates in a FITS file on disk.

S6 also utilizes multi-beam coincidence RFI rejection. Individual hits from one beam are checked for coincidence hits in the other beams. If hits are found within 25,000 frequency channels (corresponding to $\sim 20.9 \mathrm{kHz}$ ) on either side of the event's channel, and five samples (corresponding to $5.99 \mathrm{~s}$ ) on either side in time, across two or more beams, they are flagged as RFI. These frequency and time spans, and our S/ $\mathrm{N}$ of 20, are chosen empirically based on the prevailing average RFI conditions on site and our need to ensure that potential astrophysical signals are retained as hits. Assuming an exponential distribution of hit $\mathrm{S} / \mathrm{N}$ giving us $n_{\nu} n_{\mathrm{t}} n_{\mathrm{b}} n_{\mathrm{p}} e^{-20}$ events, where $n_{\nu}$ is the number of channels in our range of interest, $n_{\mathrm{t}}$ is the number of time samples, $n_{\mathrm{b}}$ is the number of beams, and $n_{\mathrm{p}}$ is the number of polarizations, for an $\mathrm{S} / \mathrm{N}$ of 20 , we expect to detect $\sim 0.01$ events in each block used in the coincidence rejection comparison. In reality, however, the statistics are dominated by RFI, and the actual number of detected events is much larger. Figure 4 shows the performance of this RFI rejection technique, applied to one polarization in one pipeline instance. We note that these plots reflect a work in progress, and additional techniques necessary to identify bona fide candidates are discussed elsewhere (V. Gajjar et al. 2017, in preparation).

In addition to the signal processing software that runs on the compute nodes, the S6 HPC system maintains a Redis keyvalue store on the head node. This is a database that is constantly updated with the status of the telescope, read from the Arecibo observatory's network, through a separate network switch (termed "AONET switch" in Figure 1). The information maintained in the database includes the receiver in use, IF frequencies, and pointing and timing information, among others.

As part of deployment and commissioning, we conducted various tests to verify the functioning of the system. The primary end-to-end test involved injecting a test signal with a bandwidth of $<0.8 \mathrm{~Hz}$ into the IF and recovering that signal at the expected level in the output data.

\subsection{ALFABURST Software}

The ALFABURST HPC cluster is very similar to that of S6. It is made up of one head node and four compute nodes. The main differences are that each compute node is equipped with a single Mellanox MCX312A-XCBT $10 \mathrm{GbE}$ NIC that receives UDP packets from the $10 \mathrm{GbE}$ switch, and uses NVIDIA GeForce GTX TITAN GPU cards that have a larger memory than those used in S6, necessitated by ALFABURST signal processing requirements.

The ALFABURST head node queries the S6 Redis database at a cadence of once per minute, checking which receiver is at the focus. When an observer selects the ALFA receiver, the corresponding value is updated in the Redis database. This is detected by ALFABURST and data acquisition is initiated. While observation is in progress, the head node continues to query the database for changes to telescope state. Data acquisition is terminated when ALFA stops being the selected receiver.

The compute nodes run the ALFABURST software data acquisition pipeline instances. Three of the compute nodes process data from two ALFA beams each, and therefore, run two instances of the software. The remaining node processes the seventh ALFA beam, and runs a single instance of the pipeline. The software architecture follows a client-server model, where the server receives incoming data from the $10 \mathrm{GbE}$ NIC, fills data corresponding to missing packets with zeros, and forwards the data to the client. The client is modular by design, with each module handling one logical signal processing stage. The ALFABURST data transport framework and signal processing system ${ }^{22}$ are based on the ARTEMIS fast transient search software developed for a recently concluded survey at the UK station of the LOFAR telescope (Karastergiou et al. 2015). Even though the software serves the Karastergiou et al. (2015) survey sufficiently, the specifications of ALFABURST are much more stringent, with a larger number of channels and a much larger bandwidth. The software in its current form is not designed to process the entire ALFA bandwidth at the native time resolution provided by the FPGA gateware. We therefore process only a bandwidth of $56 \mathrm{MHz}$, and integrate the incoming spectra with a resulting time resolution of $256 \mu \mathrm{s}$. We note that the narrowest known FRB has a width of $350 \mu$ s (Petroff et al. 2016), so the choice of time resolution is reasonable in this regard.

\footnotetext{
${ }^{22}$ The software is available upon request.
} 


\begin{tabular}{|c|c|c|c|c|c|c|c|c|c|}
\hline & 0 & 1 & 2 & 3 & 4 & 5 & 6 & 7 & $\leftarrow$ Byte \\
\hline Header & \multicolumn{6}{|c|}{ Integration count } & $S$ & $B$ & \\
\hline Sample 0 & \multicolumn{2}{|c|}{$\operatorname{Re}(X X *)$} & \multicolumn{2}{|c|}{$\operatorname{Re}(Y Y *)$} & \multicolumn{2}{|c|}{$\operatorname{Re}(X Y *)$} & \multicolumn{2}{|c|}{$\operatorname{Im}(X Y *)$} & $\leftarrow$ Channel $1024 \times S+0$ \\
\hline Sample 1 & \multicolumn{2}{|c|}{$\operatorname{Re}(X X *)$} & \multicolumn{2}{|c|}{$\operatorname{Re}(Y Y *)$} & \multicolumn{2}{|c|}{$\operatorname{Re}(X Y *)$} & \multicolumn{2}{|c|}{$\operatorname{Im}(X Y *)$} & $\leftarrow$ Channel $1024 \times S+1$ \\
\hline$\cdots$ & \multicolumn{2}{|c|}{$\cdots$} & \multicolumn{2}{|c|}{$\ldots$} & \multicolumn{2}{|c|}{$\cdots$} & \multicolumn{2}{|c|}{$\cdots$} & $\ldots$ \\
\hline Sample 1023 & \multicolumn{2}{|c|}{$\operatorname{Re}(X X *)$} & \multicolumn{2}{|c|}{$\operatorname{Re}(Y Y *)$} & \multicolumn{2}{|c|}{$\operatorname{Re}(X Y *)$} & \multicolumn{2}{|c|}{$\operatorname{Im}(X Y *)$} & $\leftarrow$ Channel $1024 \times S+1023$ \\
\hline Footer & \multicolumn{4}{|c|}{ CRC } & \multicolumn{4}{|c|}{0} & \\
\hline
\end{tabular}

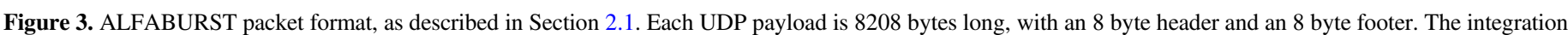

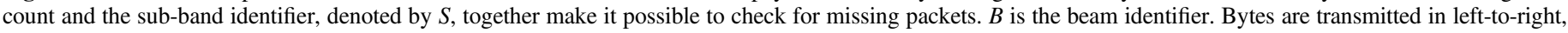
top-to-bottom order, i.e., in network byte order.

The first stage in the signal processing pipeline is the computation of full-Stokes values from the pseudo-Stokes values in the packets following

$$
\begin{aligned}
I & =X X^{*}+Y Y^{*}, \\
Q & =X X^{*}-Y Y^{*}, \\
U & =2 \operatorname{Re}\left(X Y^{*}\right), \text { and } \\
V & =-2 \operatorname{Im}\left(X Y^{*}\right) .
\end{aligned}
$$

The search process requires only the total power (Stokes $I$ ), but it is worth saving the other Stokes parameters for polarization studies of any detected FRB. In our current implementation, we do not store the other Stokes parameters, but this will be supported in future versions of the software. Stokes computation is followed by the signal processing stages involved in searching for FRBs. The following discussion briefly describes these signal processing steps; for details, we refer the reader to Karastergiou et al. (2015).

Since searching for FRBs involves correcting for the unknown dispersion delay introduced by the ISM, the major signal processing operation involves dedispersing the data over a range of trial DMs. We call this process the dedispersion transform, converting dynamic spectra (frequency versus time versus power) to a set of dedispersed time series (DM versus time versus power). Dedispersion involves summing the data over all frequency channels, so it is important to remove the data of strong RFI that would otherwise result in a large number of false positives. Accordingly, the data goes through the RFI clipper module. The RFI clipper implements an adaptive thresholding algorithm (Karastergiou et al. 2015) that takes into account the non-flat nature of the bandpass and the time-varying baseline, and normalizes the output to have a mean of 0 and a standard deviation of 1 .

Following RFI removal and spectrum normalizaton, the data undergoes the dedispersion transform. Being the most compute-intensive operation, this is run on the GPU, and is implemented in Compute Unified Device Architecture. ${ }^{23}$ The dedispersion module is based on the Astro-Accelerate code developed by Armour et al. (2012). The dedispersion transform is performed on data resident in buffers 32768 samples long. At the currently used time resolution of $256 \mu \mathrm{s}$, this corresponds to a duration of $\sim 8.4 \mathrm{~s}$. Temporal continuity across buffers is maintained by reusing the last $n_{\text {maxshift }}$ time samples from the previous buffer, where $n_{\text {maxshift }}$ is the number of time samples

\footnotetext{
${ }^{23}$ http://www.nvidia.com/object/cuda_home_new.html
}

in the lowest frequency channel to be "shifted," corresponding to the maximum DM. If downstream processing results in a detection, data from the buffer that contains the pulse is saved to the disk for later inspection.

We perform an incoherent dedispersion search over a DM range of $[0,2560] \mathrm{cm}^{-3} \mathrm{pc}$. The maximum DM among all known FRBs is $\sim 1629 \mathrm{~cm}^{-3}$ pc (Champion et al. 2016), so our upper limit is a reasonable choice. Even though the optimal DM step size is non-uniform across the DM range of interest (Cordes \& McLaughlin 2003), it is simpler to implement a fixed step size as is done in Armour et al. (2012), thereby oversampling the DM space at larger values, and, depending on the step size, possibly undersampling the DM space at smaller values. Undersampling the DM space implies the use of trial DMs that are offset from the true values, and this leads to pulse broadening, resulting in a loss in sensitivity. The smallest step size in an incoherent dedispersion search required to minimize this loss in sensitivity is

$$
\delta \mathrm{DM}=1.205 \times 10^{-7} t_{\mathrm{samp}} \nu^{3} / \Delta \nu \mathrm{cm}^{-3} \mathrm{pc},
$$

where $t_{\text {samp }}$ is the sampling interval in ms, $\nu$ is the center frequency in $\mathrm{MHz}$, and $\Delta \nu$ is the bandwidth in $\mathrm{MHz}$ (Lorimer $\&$ Kramer 2005). For a nominal center frequency of $1375 \mathrm{MHz}$, our experimental setup yields $\delta \mathrm{DM} \approx 1.4 \mathrm{~cm}^{-3} \mathrm{pc}$. As our fixed step size, we have chosen $1 \mathrm{~cm}^{-3} \mathrm{pc}$, which results in no loss in sensitivity.

The dedispersion transform step is followed by smoothing of the dedispersed time series. Each time series is decimated by factors of $2-16$, in powers of 2 . This is a matched filtering operation meant to increase the sensitivity of the search to pulses of varying widths. We note that the decimation is performed block-wise in the current implementation, as opposed to using a running window. Compared to doing true matched filtering, i.e., using a running window, this has the effect of reducing the net sensitivity by a factor of $\sqrt{2}$ (Keane \& Petroff 2015). All time series are then subject to a sensitivity threshold of 10 times the noise rms. We do not explicitly compute the rms of the data. By design, the RFI clipper outputs spectra with a standard deviation of 1 , so we take the rms to be the square root of the number of channels that are summed during dedispersion. Events that cross the threshold are saved to a candidate list that is written to disk, along with the RFI-removed filterbank data corresponding to the data buffer the event was found in. Figure 5 is a schematic of the aforementioned operations.

Observations are followed by the generation of diagnostic plots of threshold-crossing event $\mathrm{S} / \mathrm{Ns}$ as a function of time and DM. In the current scheme, plots are automatically 

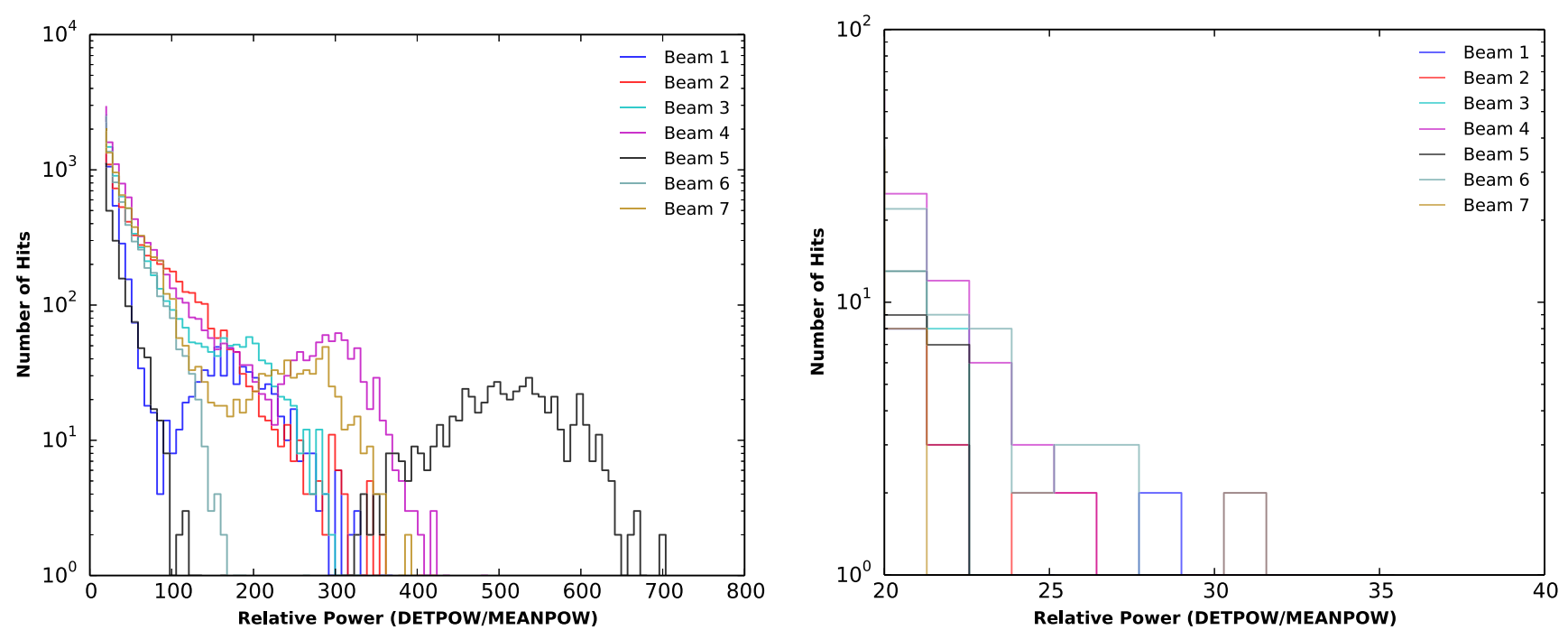

Figure 4. Histograms of the ratio of detected power ("DETPOW") and mean power ("MEANPOW") in a coincidence rejection comparison block (see Section 2.2) of typical SERENDIP VI multi-beam data. This particular data comes from one of the system's $34 \mathrm{MHz}$ sub-bands processed by a single pipeline instance, centered at $1252 \mathrm{MHz}$, spanning $\sim 800 \mathrm{~s}$. The left panel shows the power distribution before RFI rejection, and the right panel shows the power distribution after the application of multi-beam RFI rejection that removes events that are found in two or more beams that are similar in frequency and time.

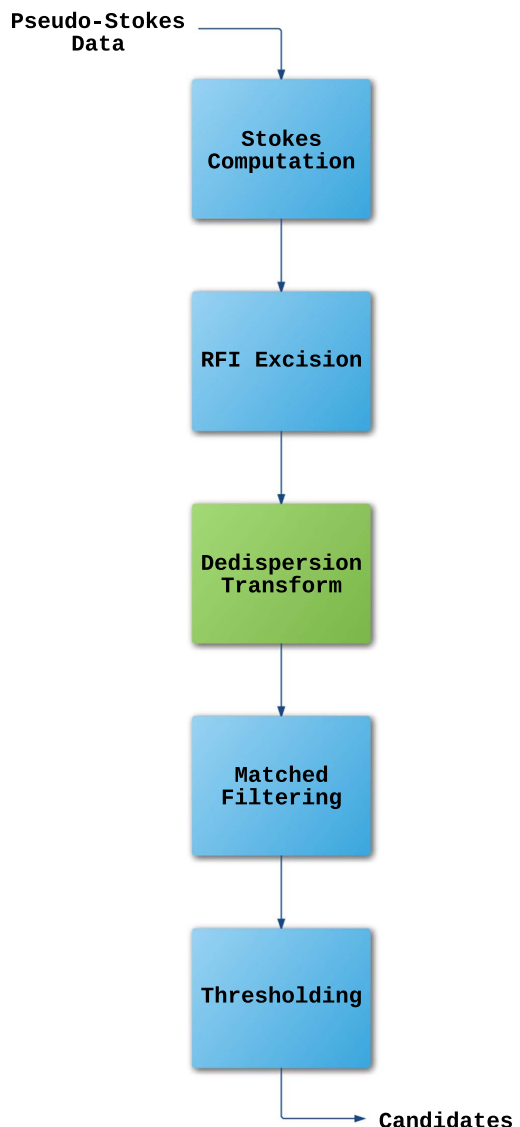

Figure 5. Signal processing stages of the ALFABURST software pipeline. The dedispersion transform is implemented on GPUs.

generated once a day, around noon local time. This makes plots available within a few hours of recording of the signal. Web pages containing these plots are automatically generated and made available using a web server. ${ }^{24}$ Beyond this stage, data analysis is manual in nature. Plots are inspected visually, and

\footnotetext{
${ }^{24}$ http://www.naic.edu/ alfafrb/
}

interesting events are followed up by examining the saved filterbank data. For pulses that are seen in the filterbank data, we compare the pointing information and DM to the entries in the ATNF Pulsar Catalog ${ }^{25}$ (Manchester et al. 2005) to check whether they correspond to known pulsars.

Commissioning tests of the system were conducted from 2015 March to August. Figure 6 shows the results of one of our commissioning observations, wherein we observed the pulsar B0611+22 in beam 1. We obtained detections whose S/Ns peaked around $97 \mathrm{~cm}^{-3} \mathrm{pc}$, as expected for the test pulsar. To verify the functionality of the system further, we compared the number of events we detected to that obtained by applying the same $\mathrm{S} / \mathrm{N}$ threshold to a time series that was dedispersed using the SIGPROC ${ }^{26}$ software, which is a standard pulsar data processing package, yielding a match.

\section{Commensal Surveys}

\subsection{Sky Coverage}

The S6 and ALFABURST surveys piggyback on ongoing Arecibo surveys, specifically the PALFA and AGES surveys. PALFA is a survey for pulsars and fast transients (Cordes et al. 2006), and has so far resulted in the discovery of 145 pulsars and one FRB (Spitler et al. 2014; Lazarus et al. 2015). Being a pulsar survey, PALFA emphasizes coverage of the Galactic plane. PALFA pointings are toward the inner Galaxy $\left(32^{\circ} \lesssim l \lesssim 77^{\circ} ; \quad|b|<5^{\circ}\right)$ and the outer Galaxy $\left(168^{\circ} \lesssim \tilde{l} \lesssim 214^{\circ} ;|b|<5^{\circ}\right)$, with dwell times of $268 \mathrm{~s}$ and $180 \mathrm{~s}$, respectively. PALFA has been allocated $230 \mathrm{hr}$ over the coming year.

AGES is an extragalactic HI survey, observing multiple fields spread in right ascension across the northern sky (Auld et al. 2006). Most fields are about $5 \times 4 \mathrm{deg}^{2}$ in size. Each pointing in the survey has a dwell time of $300 \mathrm{~s}$. AGES is expected to observe for about $350 \mathrm{hr}$ over the coming year (R. Minchin 2016, private communication). AGES pointings

\footnotetext{
25 http://www.atnf.csiro.au/people/pulsar/psrcat/

${ }^{26} \mathrm{http}: / /$ sigproc.sourceforge.net/
} 


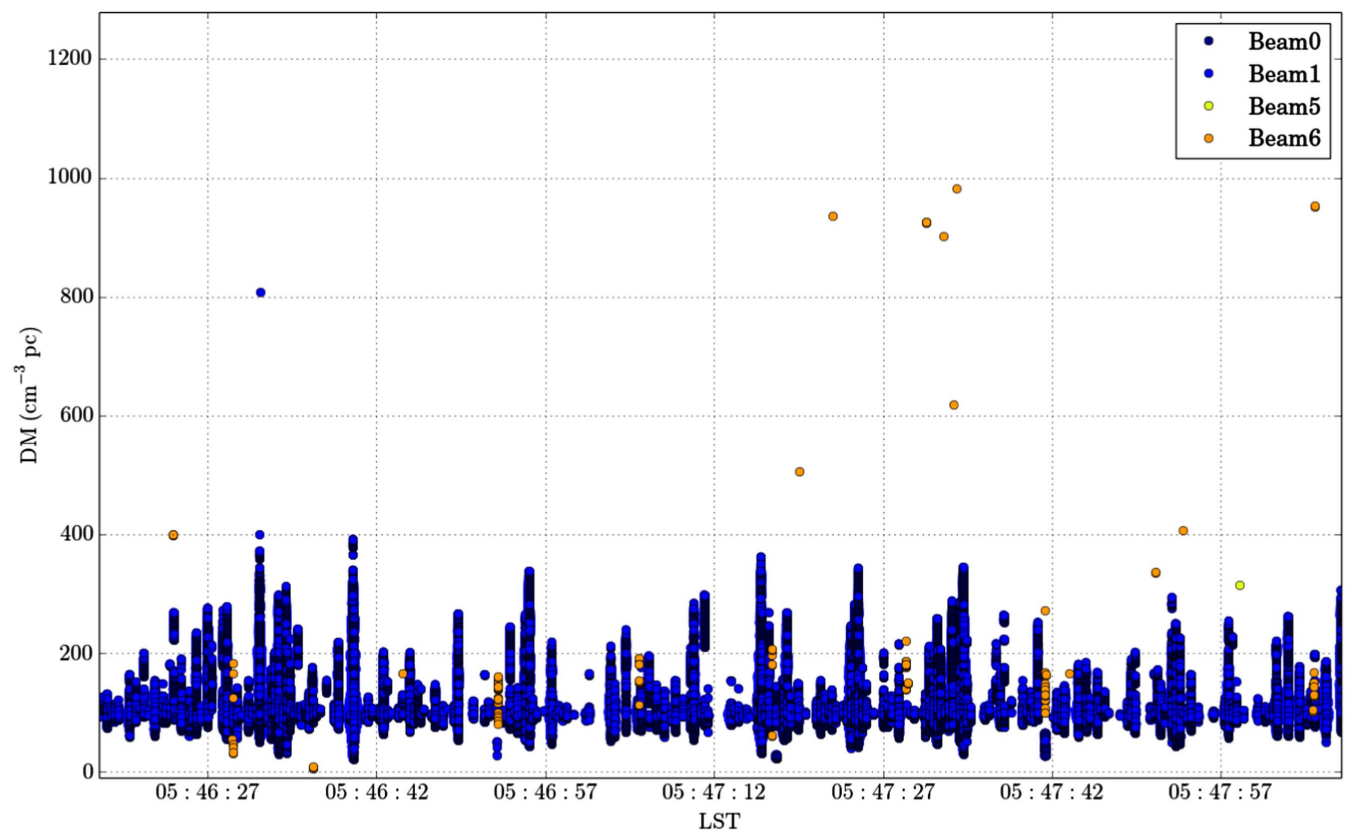

Figure 6. ALFABURST commissioning test results for an observation of the pulsar B0611+22. The pointing was such that the pulsar was in beam 1 of ALFA. The markers represent events whose $\mathrm{S} / \mathrm{Ns}$ crossed our detection threshold. The clusters of detections are centered around a DM of $97 \mathrm{~cm}^{-3} \mathrm{pc}$, as expected for this pulsar.

are mostly away from the Galactic plane, and therefore are conducive to a search for extragalactic FRBs.

\subsection{SERENDIP VI Sensitivity}

For a narrow-band signal in a single polarization, the minimum detectable flux, in $\mathrm{W} \mathrm{m}^{-2}$, of a signal that has a width less than the channel bandwidth $\Delta f \mathrm{~Hz}$ is given by

$$
F=\sigma S_{\text {sys }} \sqrt{\frac{\Delta f}{t}}
$$

where $\sigma$ is the threshold $\mathbf{S} / \mathrm{N}, S_{\text {sys }}$ is the system equivalent flux density (SEFD) in Jy, and $t$ is the integration time in $\mathrm{s}$.

We use a detection threshold of $20 \sigma$, which has been determined empirically, based on the RFI environment at the site. Given that the boresight SEFD of ALFA is $2.73 \mathrm{Jy}$, for our threshold $\mathrm{S} / \mathrm{N}$, for an integration time of $1.198 \mathrm{~s}$, with a channel bandwidth of $\sim 0.8 \mathrm{~Hz}$, the minimum detectable flux is $\sim 4.6 \times 10^{-25} \mathrm{~W} \mathrm{~m}^{-2}$, or $\sim 55 \mathrm{Jy}$ across a channel.

As an example of a transmitter, we consider the case of the $2380 \mathrm{MHz}$ transmitter of the Arecibo Planetary Radar, which is frequently used to determine the orbits of near-Earth asteroids. It has an EIRP of $\sim 2 \times 10^{13} \mathrm{~W}$. Our sensitivity is high enough to detect similar transmitters up to a distance of $\sim 60 \mathrm{pc}$. However, this energetics comparison is strictly for illustrative purposes. The detectability and decoding of terrestrial analogs at interstellar distances is a complex topic (see Sullivan et al. 1978) and is not addressed here.

\subsection{Alfaburst Sensitivity}

Following the radiometer equation (see, for example, Lorimer \& Kramer 2005), the threshold flux density of a single pulse search,

$$
S_{\min }=\frac{\sigma S_{\mathrm{sys}}}{\sqrt{n_{\mathrm{p}} \Delta f W}},
$$

where $\sigma$ is the threshold $\mathrm{S} / \mathrm{N}, S_{\text {sys }}$ is the SEFD, $n_{\mathrm{p}}$ is the number of polarizations, $\Delta f$ is the bandwidth in $\mathrm{MHz}$, and $W$ is the pulse width in ms. In the absence of RFI, the choice of $\mathrm{S} / \mathrm{N}$ threshold for a single pulse search is rather straightforward. If we assume Gaussian statistics, the number of events crossing the threshold $\sigma$ is

$$
N(>\sigma) \approx 2 n_{\mathrm{samp}} \theta n_{\mathrm{DM}}
$$

where $n_{\text {samp }}$ is the number of time samples, $n_{\mathrm{DM}}$ is the number of DM channels, and $\theta$ is the probability of occurrence of a sample with peak above $\sigma$ (Cordes \& McLaughlin 2003). A reasonable value of $\sigma$ for one ALFABURST buffer, i.e., about $8.4 \mathrm{~s}$, such that the number of events due to noise alone is 1 , comes out to be 5.7. In practice, however, RFI poses a significant problem, especially at Arecibo-both due to the noisy environment and the high sensitivity of the telescopeand requires us to choose a threshold that is much higher. We have empirically determined 10 as our optimal $\mathrm{S} / \mathrm{N}$ threshold, eliminating a large fraction of spurious events, while minimizing the likelihood of missing a potential astrophysical signal. After applying this threshold, we discard less than 5 per cent of the observed time span to RFI.

For an FRB with a width of $1 \mathrm{~ms}$, located at the central beam boresight of ALFA, our sensitivity is $\sim 2.6 \mathrm{mJy}$. All known FRBs have observed peak flux densities ranging upwards of $\sim 200 \mathrm{mJy}$, therefore, in spite of not utilizing the whole ALFA band, our sensitivity is reasonable.

\subsubsection{Event Rates}

Given that the ALFABURST survey is a commensal survey that piggybacks on multiple surveys intended for multiple applications, with each survey observing a different part of the sky for different amounts of time, and the fact that FRB event rates and Galactic latitude dependence are not well constrained, it is challenging to come up with a rigorous expectation of the 
number of detections. Therefore, we follow a naive approach, merely extrapolating from the expected usage duration and instantaneous field-of-view (FoV) of ALFA, and the event rate computed by Scholz et al. (2016) based on the Arecibo FRB detection. ALFA is expected to be used by PALFA and AGES for $\sim 580 \mathrm{hr}$ over the next year. It has an instantaneous FoV of $\sim 0.02$ sq. deg. within the full-width half-maximum. Scholz et al. (2016) compute an event rate of $5.08_{-4.81}^{+17.78} \times 10^{4}$ sky $^{-1}$ day $^{-1}$ for bursts with flux density above $57 \mathrm{mJy}$. Together, this leads to an expectation of between zero and five such FRB detections in the coming year.

\section{Conclusion}

We have designed, built, and deployed an automated, commensal, realtime, multi-science backend for the Arecibo telescope that conducts two surveys simultaneously. S6 is conducting a survey for technologically advanced life, whereas ALFABURST is conducting a survey for fast radio transients.

Future work for S6 involves replicating the system at other observatories. We are in the process of building a similar system for the Green Bank Telescope. Part of the data from both Arecibo and Green Bank will eventually be sent out over the SETI@home ${ }^{27}$ citizen science distributed computing system for processing.

Future work for ALFABURST involves supporting the whole ALFA bandwidth of $300 \mathrm{MHz}$, the native FPGA time resolution of $128 \mu \mathrm{s}$, and searching a larger range of DMs. The fact that ALFA has multiple beams can be used for the coincidence rejection of RFI-if a signal appears in all seven beams, it is likely that it is of terrestrial origin. We also intend to support the $327 \mathrm{MHz}$ receiver whose usage is more than that of ALFA, letting us not only increase the survey time, but also perform a realtime, commensal survey for FRBs at a relatively less explored part of the spectrum. The results of a $327 \mathrm{MHz}$ survey would enable us to constrain the spectral index of FRBs, similar to what was done with the non-detection at $145 \mathrm{MHz}$ by Karastergiou et al. (2015). Longer-term goals include reducing the latency involved in the generation of diagnostic plots, automatic classification of candidate signals, and implementing a mechanism for triggering telescopes that operate at lower frequencies, following a detection.

We thank the anonymous referee for feedback that greatly improved the manuscript. We thank the staff of the Arecibo Observatory, in particular, Hector Hernandez, Robert Minchin, Mike Nolan, Phil Perillat, Luis Quintero, Joan Schmelz, Arun Venkataraman, and Dana Whitlow for support with instrument deployment and commissioning tests. We also acknowledge the cooperation of the PALFA and AGES consortia. J.C., A.K., and W.A. thank the Leverhulme Trust for support with instrument development. D.R.L. and M.A.M. were supported through NSF Award \#1458952. Some of the ALFABURST hardware was purchased with funds from the WVU Eberly College of Arts and Sciences and the WVU Department of Physics and Astronomy. We also thank the NASA ASTID Program, the John Templeton Foundation, and the Breakthrough Prize Foundation for their support. We thank Eric Korpela for useful discussions.

Facility: Arecibo.

\section{References}

Armour, W., Karastergiou, A., Giles, M., et al. 2012, in ASP Conf. Ser. 461, Astronomical Data Analysis Software and Systems XXI, ed. P. Ballester, D. Egret, \& N. P. F. Lorente (San Francisco, CA: ASP), 33

Auld, R., Minchin, R. F., Davies, J. I., et al. 2006, MNRAS, 371, 1617

Bowyer, S., Werthimer, D., Donnelly, C., et al. 1993, in ASP Conf. Ser. 47, Third Decennial US-USSR Conference on SETI, ed. G. S. Shostak (San Francisco, CA: ASP), 269

Bowyer, S., Zeitlin, G., Tarter, J., Lampton, M., \& Welch, W. J. 1983, Icar, 53,147

Champion, D. J., Petroff, E., Kramer, M., et al. 2016, MNRAS, 460, L30

Chatterjee, S., Law, C. J., Wharton, R. S., et al. 2017, arXiv:1701.01098

Cocconi, G., \& Morrison, P. 1959, Natur, 184, 844

Cohen, R. J., Downs, G., Emerson, R., et al. 1987, MNRAS, 225, 491

Cordes, J. M., Freire, P. C. C., Lorimer, D. R., et al. 2006, ApJ, 637, 446

Cordes, J. M., \& McLaughlin, M. A. 2003, ApJ, 596, 1142

Drake, F. D. 1961, PhT, 14, 40

DuPlain, R., Ransom, S., Demorest, P., et al. 2008, Proc. SPIE, 7019, 70191D

Falcke, H., \& Rezzolla, L. 2014, A\&A, 562, A137

Hewish, A., Bell, S. J., Pilkington, J. D. H., Scott, P. F., \& Collins, R. A. 1968, Natur, 217, 709

Karastergiou, A., Chennamangalam, J., Armour, W., et al. 2015, MNRAS, 452, 1254

Keane, E. F., Johnston, S., Bhandari, S., et al. 2016, Natur, 530, 453

Keane, E. F., \& Petroff, E. 2015, MNRAS, 447, 2852

Lazarus, P., Brazier, A., Hessels, J. W. T., et al. 2015, arXiv:1504.02294

Loeb, A., Shvartzvald, Y., \& Maoz, D. 2014, MNRAS, 439, L46

Lorimer, D. R., Bailes, M., McLaughlin, M. A., Narkevic, D. J., \& Crawford, F. 2007, Sci, 318, 777

Lorimer, D. R., \& Kramer, M. 2005, Handbook of Pulsar Astronomy (Cambridge: Cambridge Univ. Press)

Lyutikov, M., Burzawa, L., \& Popov, S. B. 2016, arXiv:1603.02891

Macquart, J.-P., Bailes, M., Bhat, N. D. R., et al. 2010, PASA, 27, 272

Manchester, R. N., Hobbs, G. B., Teoh, A., \& Hobbs, M. 2005, AJ, 129, 1993

Masui, K., Lin, H.-H., Sievers, J., et al. 2015, Natur, 528, 523

McLaughlin, M. A., Lyne, A. G., Lorimer, D. R., et al. 2006, Natur, 439, 817

Mottez, F., \& Zarka, P. 2014, A\&A, 569, A86

Petroff, E., Barr, E. D., Jameson, A., et al. 2016, arXiv:1601.03547

Popov, S. B., \& Postnov, K. A. 2013, arXiv:1307.4924

Scholz, P., Spitler, L. G., Hessels, J. W. T., et al. 2016, arXiv:1603.08880

Siemion, A. P. V., Demorest, P., Korpela, E., et al. 2013, ApJ, 767, 94

Spitler, L. G., Cordes, J. M., Hessels, J. W. T., et al. 2014, ApJ, 790, 101

Spitler, L. G., Scholz, P., Hessels, J. W. T., et al. 2016, Natur, 531, 202

Sullivan, W. T., III, Brown, S., \& Wetherill, C. 1978, Sci, 199, 377

Taylor, A. R., \& Salter, C. J. 2010, in ASP Conf. Ser. 438, The Dynamic Interstellar Medium: A Celebration of the Canadian Galactic Plane Survey, ed. R. Kothes, T. L. Landecker, \& A. G. Willis (San Francisco, CA: ASP), 402

Thornton, D., Stappers, B., Bailes, M., et al. 2013, Sci, 341, 53

Totani, T. 2013, PASJ, 65, L12

Wayth, R. B., Tingay, S. J., Deller, A. T., et al. 2012, ApJL, 753, L36

Welch, J., Backer, D., Blitz, L., et al. 2009, IEEEP, 97, 1438

Woods, A. 2010, MA thesis, Univ. Cape Town

27 http://setiathome.berkeley.edu/ 